\title{
The cerebellum is associated with 2-year prognosis in patients with high-frequency migraine
}

Hung-Yu Liu ${ }^{1,2}$, Pei-Lin Lee ${ }^{3}$, Kun-Hsien Chou ${ }^{3,4}$, Kuan-Lin Lai ${ }^{1,2}$, Yen-Feng Wang ${ }^{1,2}$, Shih-Pin Chen ${ }^{1,2,4,5}$, Wei-Ta Chen ${ }^{1,2,4^{*}}$ and Shuu-Jiun Wang ${ }^{1,2,4^{*}}$

\begin{abstract}
Background: The increase of headache frequency is associated with higher headache related disability and lower quality of life in patients with migraine. However, the pathophysiology of migraine progression, persistence, or remission is elusive. The purpose of this study is to identify the brain signatures that are predictive of the long-term outcomes among patients with high-frequency migraine (HFM: 10-30 headache days/month).

Methods: We prospectively enrolled patients with HFM and healthy controls and collected their baseline clinical profiles and brain-MRI data at first visit. We longitudinally followed the patients and determined their outcomes at 2-year follow-up. Good outcome was defined as $\geq 50 \%$ reduction of baseline headache days and poor outcome was defined as reduction $<50 \%$ or frequency increase. Voxel-based morphometry was used to study gray matter volume (GMV), and structural covariance was used to investigate structural connectivity.

Results: Among 56 patients with HFM, 37 had good outcome and 19 poor outcome. Compared to the healthy controls ( $n=37)$, patients with poor outcome had decreased GMV over the left posterior cingulate gyrus, and increased GMV over the bilateral cerebellum and the right precentral gyrus. Further, patients with poor outcome had greater GMV over the right and the left cerebella compared to patients with good outcome, and the GMVs of the cerebella were correlated to 2-year headache frequencies (right: $r=0.38, P=0.005$; left: $r=0.35, P=0.009$ ). Structural connectivity were increased between the cerebellum and the cuneus, the calcarine cortex, and the temporal lobe, respectively, in patients with poor outcome, and was decreased between the cerebellum and the prefrontal cortex in patients with poor outcome. The structural covariance integrities between the right cerebellum and the right cuneus were correlated to 2-year headache frequencies $(r=0.36, P=0.008)$.
\end{abstract}

Conclusions: Structural volume and connectivity changes of the cerebellum may underlie headache persistence in patients with HFM.

Keywords: Cerebellum, Migraine, High frequency, Outcome, MRI

\footnotetext{
*Correspondence: wtchen71@gmail.com; wtchen@vghtpe.gov.tw; sjwang@vghtpe.gov.tw

'Department of Neurology, Neurological Institute, Taipei Veterans General Hospital, No. 201, Sec. 2 Shih-Pai Rd, Taipei, Taiwan

Full list of author information is available at the end of the article
}

(c) The Author(s). 2020 Open Access This article is licensed under a Creative Commons Attribution 4.0 International License, which permits use, sharing, adaptation, distribution and reproduction in any medium or format, as long as you give appropriate credit to the original author(s) and the source, provide a link to the Creative Commons licence, and indicate if changes were made. The images or other third party material in this article are included in the article's Creative Commons licence, unless indicated otherwise in a credit line to the material. If material is not included in the article's Creative Commons licence and your intended use is not permitted by statutory regulation or exceeds the permitted use, you will need to obtain permission directly from the copyright holder. To view a copy of this licence, visit http://creativecommons.org/licenses/by/4.0/ The Creative Commons Public Domain Dedication waiver (http://creativecommons.org/publicdomain/zero/1.0/) applies to the data made available in this article, unless otherwise stated in a credit line to the data. 


\section{Introduction}

Migraine is a recurrent headache disorder, with its attack frequency fluctuating during the disease course [1-3]. Some patients with episodic migraine may transform to chronic migraine (CM: headache days $\geq 15 /$ month and migraine days $\geq 8$ /month for more than 3 months). Fortunately, most of the patients with CM have headache remission to episodic form with time. However, some patients with CM persistently suffer from frequent migraine attacks, with their headache-related burdens influencing their occupations, as well as their social and family functioning [4].

The pathophysiology of migraine progression, persistence, or remission is yet elusive. Current evidences have shown some risk factors for migraine progression or persistence. Baseline high-frequency headaches, medication overuse, and depression are associated with migraine progression [5, 6], while medication overuse and chronic musculoskeletal complaints are predictors of chronic headache persistence [2]. In the field of neuroimage, altered brain volumes and activities have been demonstrated in patients with CM compared to the patients with episodic migraine or the healthy controls (HC). However, whether such changes reflect the current status of frequent attacks, the longitudinal consequences of migraine attacks, or are pathogenic and involved in headache chronification is not clearly understood [7-9].

This study aims to explore the neuroimage signatures related to long-term prognosis in patients with migraine with frequent attacks. High-frequency episodic migraine (10-14 headache days/month) is the most susceptible group among episodic migraine to evolve to $\mathrm{CM}$, and is as disabling as CM [10-12]. Therefore, we considered highfrequency episodic migraine in a continuum with CM, and investigated both types as a single entity, termed "high-frequency migraine" (HFM; 10-30 headache days/ month). We prospectively recruited patients with HFM, obtained their clinical profiles and brain-MRI at baseline, and longitudinally followed-up their headache status after 2 years. The outcomes of the patients were determined by their follow-up 2-year headache frequency. We explored the clinical and neuroimaging data between patients with HFM with different outcomes. We sought to identify the brain structures that are pathogenic to chronic headache with frequent attacks.

\section{Methods}

\section{Participants}

Headache specialists at the Headache Clinic of the Taipei Veterans General Hospital prospectively surveyed patients aged 20-60 years with newly-diagnosed migraine from May 2011 to Jan 2017. The diagnosis of migraine followed the International Classification of Headache Disorders-3 criteria [13]. We included patients with high frequency episodic migraine and CM, and excluded patients with severe depression, i.e., Hospital Anxiety and Depression Scale-Depression (HADS-D) score $\geq 15$, or those with comorbid medication overuse, i.e., using abortive headache medication for more than 10 or 15 days per month [14]. We recruited age- and sex-matched HC for comparison.

All participants were right-handed, denied any history of systemic or major neurological diseases, and presented with normal neurologic examinations. We encrypted information that could potentially expose individual identity.

\section{Standard protocol approvals, registrations, and patient consents}

All participants completed informed consent forms after receiving a complete explanation of the study. The Institutional Review Board of our hospital approved the study protocol.

\section{Study design}

All participants filled out a semi-structured questionnaire at their first visit to obtain demographic information and headache profiles. We defined headache frequency as the average number of headache days per month in the last 3 months and duration of headache history as the duration in years between the first migraine episode and the date of the first visit to our headache clinic. We evaluated mood based on severity of depression and anxiety using the HADS [14] and functional disability caused by migraine using the Migraine Disability Assessment Scale (MIDAS) [15].

Each participant underwent scheduled MRI during the interictal period, defined as absence of acute migraine attack within 2 days prior and subsequent to the date of image acquisition. We rescheduled the scanning session if there was an acute migraine episode during this period or use of analgesics, triptans, or ergots for any reason within $48 \mathrm{~h}$ prior to scanning.

\section{Follow-up}

After the MRI scanning, all included patients were treated at our headache clinic by headache specialists according to their clinical experience. Two years after the first visit, physician interviewed the patients by telephone to assess their migraine status and headache frequency within the last 3 months. Based on the comparison of current headache frequency to that at first visit, we defined good outcome as $\geq 50 \%$ reduction in baseline headache frequency and poor outcome as lower than $50 \%$ reduction in headache frequency or as frequency increase. Medical records were also reviewed to assess the use of migraine medication in these patients. Among the 56 patients included in our analysis, 48 were treated with both migraine prophylactic (including topiramate, propranolol, metoprolol, flunarizine, valproic acid, tricyclic acid, or amitriptyline) and abortive medications (sumatriptan or non- steroidal anti- 
inflammatory drugs), while the other 8 patients were treated with abortive medications only.

\section{MRI data acquisition}

We used the same 3.0 T GE Discovery MR750 scanner (General Electric Healthcare, Milwaukee, WI, USA) at Taipei Veterans General Hospital to acquire all data with a standard eight-channel phase array head coil.

We acquired the T1-weighted anatomical scans with two different acquisition pulse sequences including: 1) an inversion recovery prepared fast spoiled gradient-recalled echo sequence (IR-FSPGR) and 2) an IR-FSPGR-brain volume imaging (BRAVO) sequence.

Of note, 32 of 56 patients with HFM and 29 of $37 \mathrm{HC}$ in this study were also included in a dataset that was recently used to analyze hippocampal volume changes in patients with migraine [16]; these patients underwent the first acquisition pulse sequence, whereas the rest and the $\mathrm{HC}$ underwent the second acquisition pulse sequence.

The detailed imaging parameters of each protocol were as follows: repetition time/echo time/inversion time $=9.4$ (IR-FSPGR sequence) or 9.2 (IR-FSPGR-BRAVO sequence) / 4.0 (IR-FSPGR sequence) or 3.7 (IR-FSPGRBRAVO sequence) $/ 450 \mathrm{~ms}$; flip angle $=12^{\circ}$, matrix size $=$ $256 \times 256$, field of view $=256 \times 256 \mathrm{~mm}^{2}$, number of excitations $=1$, slice thickness $=1 \mathrm{~mm}$ without inter-slice gap and interpolation, and 172 (IR-FSPGR sequence) or 168 (IR-FSPGR-BRAVO sequence) axial contiguous slices. An experienced neuroradiologist visually inspected all MRI scans to exclude any organic brain disorders; no participant was excluded for brain abnormalities. Before subsequent image processing, we reoriented all T1-weighed scans using a center-of-mass approach to minimize the position difference during the data acquisition.

\section{Calculation of gray matter volume information}

To estimate individual voxel-wise gray matter volume (GMV) maps, we processed T1-weighted scans using the voxel based morphometry (VBM) [17] pipeline with Statistical Parametric Mapping 12 (SPM12, version 7487, Wellcome Institute of Neurology, University College London, UK) under the MATLAB environment (version R2015b; Mathworks, Natick, MA). We corrected individual T1-weighted scans for intensity inhomogeneities, segmented them into GM, white matter (WM), and cerebrospinal fluid (CSF); and initially rigid-aligned them to the Montreal Neurological Institute (MNI) space using the SPM12 "Segment" function. To improve tissue classification accuracy of the subcortical areas, which are highly involved in the pathophysiology of migraine, we incorporated the enhanced tissue probability maps for subcortical regions to the above tissue segmentation procedure [18]. We then used the Diffeomorphic Anatomical Registration through Exponentiated Lie algebra
(DARTEL) toolbox to generate study-specific tissue templates, by iteratively registering the ridge-aligned GM and WM segments of all study participants, and to further warp individual tissue segments to the constructed templates [19]. We modulated the individual MNI-space GM tissue segments with the corresponding DARTEL flow field to ensure that the following statistical analyses would be more sensitive to local GMV changes. Finally, we smoothed the modulated GMV maps with an isotropic Gaussian filter (full width at half maximum $=8 \mathrm{~mm}$ ) and we further excluded voxels with GM probability lower than 0.2 . We set the final spatial resolution of all GMV maps to $1.5 \mathrm{~mm}^{3}$. We estimated the individual global tissue volume and total intracranial volume (TIV $=\mathrm{GM}+\mathrm{WM}+\mathrm{CSF}$ volumes) in native $\mathrm{T} 1$ space to adjust for the effect of global brain size in the following statistical analyses.

\section{Minimization of the influence of different acquisition protocols using data harmonization modeling}

In this study, we used the ComBat harmonization approach to reduce the potential influence of different image acquisition protocols in GMV measurements [20]. ComBat was originally designed to correct for "batch effects" in genomic studies, and recent multi-site neuroimaging studies adapted this approach to remove unwanted non-biological variability while preserving meaningful associations between image variables and covariate of interests [21-23]. This approach uses a multivariate linear mixed effects regression with terms for biological variables and imaging protocols to model quantitative measurements (for example: voxel-wise GMV maps in the current study). In more detail, we included "group" as a covariate of interest to preserve potential biological trends in the data and simultaneously corrected for the effect of different acquisition protocols.

\section{Statistical analysis}

\section{Analyses of demographic and clinical data}

The descriptive data in the demographic and clinical profiles are presented as mean \pm standard deviation or numbers and percentages. We used the chi-square test to test for differences in categorical data. We used Student's t test to compare the means of normally-distributed continuous variables, and the Mann-Whitney $\mathrm{U}$ test to compare nonnormally-distributed variables, i.e., the MIDAS scores.

\section{Analyses of voxel-wise imaging-based investigations}

We used SPM12 with appropriate statistical models to perform the following voxel-wise statistical analyses. We adopted the cluster-extent statistical approach with the updated "3dFWHMx" and "3dClustSim" functions (available in the Analysis of Functional Neuroimages software, version 19.1.18; 10,000 Monte Carlo simulations with explicit GM mask) to correct for multiple comparisons across the whole-brain voxels. An initial voxel-level $P$-value $<0.005$ 
with 257 extended voxels was considered statistically significant at cluster-level family-wise error (FWE) ratecorrected P-value $<0.05$ for all voxel-wise statistical analyses (including VBM and structural covariance (SC) network analysis). The details of the statistical models are listed below.

\section{Identification of GMV alterations among patients with HFM with different outcomes and HC}

To identify the GMV difference among patients with HFM with different outcome status and the $\mathrm{HC}$, a single-factor three-level (HFM with good outcome, HFM with poor outcome, and $\mathrm{HC}$ ) analysis of covariance (ANCOVA) design was employed, with age, sex, TIV, and HADS scores entered as nuisance variables. The following contrasts were tested: HFM vs. HC, HFM with good outcome vs. HC, HFM with poor outcome vs. HC, and HFM with good outcome vs. HFM with poor outcome. We extracted, averaged, and correlated the GMV of the clusters with a significant between-group effect with headache profiles using partial Pearson's correlation to investigate the clinical relevance. We entered age, sex, TIV, and HADS scores as nuisance variables in the correlation analysis.

\section{Exploration of the changes in the SC network in patients with different outcomes}

$\mathrm{SC}$ is a volumetric correlation measurement between two brain regions. SC network analysis was recently proposed as a surrogate approach to characterizing structural connectivity profiles between distinct anatomic brain regions [24]. We generated SC networks using hypothesis-driven, seed-based correlation analysis [25] or data-driven, independent component analysis. SC networks reflect the shared covariance of brain morphologic features across the study participants and provide a quantitative means to studying cortical morphometric organization. In addition, the topology of the SC network is highly concordant with gene expression patterns and recapitulates intrinsic functional network architecture [26, 27].

We used three cerebellar regions with significant GMV alterations between the outcome groups as predefined regions of interest (ROIs) for the SC analyses. We constructed three multiple regression models for the respective ROIs to explore potential differences in the SC network between the outcome groups [28]. We integrated a group main effect term (good vs. poor outcome), a mean ROI volume main effect, and a group $\mathrm{x}$ mean ROI volume interaction term into the statistical model and included age, sex, TIV, and HADS scores to adjust for potential nuisance effects. The changes in SC integrity of the predefined ROI with identified clusters between the outcome groups could be identified by evaluating the statistical significance of the corresponding interaction term of the constructed models.

\section{Investigation of SC integrity and long-term headache frequencies}

To investigate if the changes in SC integrity between the predefined ROIs and identified clusters were correlated to 2-year headache frequencies, we applied a recentlyproposed approach to obtain a single measure that could quantify the integrity of SC for each individual [29]. We correlated the SC measures for each patient with their 2-year headache frequencies using partial Pearson's correlation after controlling for age, sex, and HADS scores.

\section{Predictive values of clinical profiles and neuroimaging data for headache outcomes}

We constructed two logistic regression models to assess the predictive values of clinical profiles only and clinical profiles with neuroimaging results for headache outcomes, respectively. We estimated the predictive values of the regression models using the area under the receiver operating characteristic curve (AUC).

We performed all statistical analyses using SPSS version 21.0 for Windows (IBM Corp., Armonk, NY), and a $P$ value $<0.05$ was regarded as significant.

\section{Results}

Demographics, clinical profiles, and headache outcomes

We enrolled 64 patients with HFM and studied their clinical headache profiles and neuroimaging data. Eight patients $(12.5 \%)$ were excluded from the analysis because they were either lost to follow-up after 2 years or could not clearly recall their current 3-month headache frequencies and provide accurate information regarding their headache days, rendering categorization of their headache outcomes difficult. We included the data of the remaining 56 patients with HFM (44 were CM and 12 were high frequency episodic migraine) in the final analyses. Of note, the 44 patients with $\mathrm{CM}$ and the 12 patients with high frequency episodic migraine did not differ in demographics, headache features, impacts, and outcome, except for a higher score of HADS-D and baseline headache frequency in those with CM (Supplement Table 1).

Patients with HFM were age- and sex-matched to the $37 \mathrm{HC}$, but they had higher HADS-A and HADS-D scores compared to the HC. Among patients with HFM, 37 had good outcome, whereas the other 19 had poor outcome. Patients with different outcomes were similar in age and sex, HADS scores, and headache characteristics, but patients with poor outcome had a borderline higher MIDAS score than did those with good outcome $(P=0.050)$. Table 1 presents the demographics and clinical profiles.

\section{GMV difference between patients with HFM and HC}

Compared to HC, patients with HFM had decreased GMV over the right supramarginal gyrus and increased GMV over the right cerebellar crus II (Table 2 and 
Table 1 Demographics, clinical profiles, and neuroimaging data of patients with HFM and HC

\begin{tabular}{|c|c|c|c|c|}
\hline & $\begin{array}{l}\mathrm{HC} \\
(n=37)\end{array}$ & $\begin{array}{l}\text { HFM } \\
(n=56)\end{array}$ & $\begin{array}{l}\text { HFM } \\
\text { Good outcome }(n=37)\end{array}$ & $\begin{array}{l}\text { HFM } \\
\text { Poor outcome }(n=19)\end{array}$ \\
\hline Age & $39.4 \pm 9.3$ & $40.3 \pm 10.5$ & $40.8 \pm 11.1$ & $39.4 \pm 9.6$ \\
\hline $\operatorname{Sex}(F / M)$ & $27 / 10$ & $43 / 13$ & $27 / 10$ & $16 / 3$ \\
\hline Aura (\%) & nil & $9(16 \%)$ & $7(18.9 \%)$ & $2(10.5 \%)$ \\
\hline Headache frequency-baseline (d/m) & nil & $19.2 \pm 7.1$ & $18.9 \pm 7.1$ & $19.7 \pm 7.4$ \\
\hline Chronic migraine & nil & $44(78.6 \%)$ & $29(78.4 \%)$ & 15 (78.9\%) \\
\hline Disease duration (years) & nil & $17.2 \pm 11.3$ & $15.7 \pm 10.2$ & $20.1 \pm 13.0$ \\
\hline Headache intensity (NRS 0-10) & nil & $6.5 \pm 1.7$ & $6.6 \pm 1.7$ & $6.3 \pm 2.0$ \\
\hline MIDAS & nil & $43.9 \pm 49.0$ & $20 \pm 50$ & $62.5 \pm 92$ \\
\hline HADS-A & $4.7 \pm 2.5^{\#}$ & $8.9 \pm 4.3$ & $9.1 \pm 4.3$ & $8.5 \pm 4.3$ \\
\hline HADS-D & $3.5 \pm 2.8^{\#}$ & $6.8 \pm 5.0$ & $6.7 \pm 5.5$ & $6.8 \pm 4.2$ \\
\hline Prophylactic drugs use & nil & $48(85.7 \%)$ & $30(81.1 \%)$ & $18(94.7 \%)$ \\
\hline Prophylactic drugs use at 2-year follow-up & nil & $16(28.6 \%)$ & $10(27 \%)$ & $6(31.6 \%)$ \\
\hline Headache frequency at 2-year follow-up (d/m) & nil & $9.0 \pm 9.5$ & $3.3 \pm 2.4^{*}$ & $20.3 \pm 7.8$ \\
\hline $\operatorname{TIV}\left(\mathrm{mm}^{3}\right)$ & $1430.8 \pm 129.6$ & $1399.8 \pm 132.5$ & $1404.7 \pm 124.6$ & $1390.2 \pm 149.7$ \\
\hline
\end{tabular}

HC healthy controls, HFM high frequency migraine, NRS numeric rating scale, MIDAS Migraine Disability Assessment, HADS-A the anxiety subscale of the Hospital Anxiety and Depression Scale, HADS-D the depression subscale of the Hospital Anxiety and Depression Scale, TIV total intracranial volume

\# denotes difference comparing patients with HFM to HC, $p<0.05$

*denotes difference comparing patients with HFM with good to poor outcomes, $p<0.05$

Fig. 1a). The volume of the right cerebellar crus II was correlated to scores of MIDAS in patients with HFM $(r=0.41, p=0.004)$. Otherwise, the two structures were not correlated to the other headache profiles in patients with HFM. There was no significant difference in GMV between patients with good outcome and HC. However, patients with poor outcome had decreased GMV over the left posterior cingulate gyrus and increased GMV

Table 2 Difference in GMV among patients with HFM with different outcomes and $\mathrm{HC}$

\begin{tabular}{llllll}
\hline \multicolumn{2}{l}{$\begin{array}{l}\text { MNl coordinates } \\
x \quad y \quad z\end{array}$} & $\begin{array}{l}\text { Voxel } \\
\text { size }\end{array}$ & Anatomical region & $\begin{array}{l}\text { Local peak } \\
\text { T-value }\end{array}$ \\
\hline HC $>$ HFM & & & \\
$50 \quad-45 \quad 41$ & 266 & Right Supramarginal Gyrus & 3.41
\end{tabular}

HC $<$ HFM

$\begin{array}{lllll}45 & -46 & -43 & 407 & \text { Right Cerebellum Crus II } \quad 3.45\end{array}$

HC > HFM with poor outcome

$\begin{array}{lllll}-14 & -20 & 38 & 287 & \text { Left Posterior Cingulate Gyrus } \quad 3.00\end{array}$

HC $<$ HFM with poor outcome

$\begin{array}{llllll}47 & -47 & -44 & 4408 & \text { Right Cerebellum Crus II } & 4.69 \\ 45 & -5 & 56 & 262 & \text { Right Precentral Gyrus } & 3.66 \\ -11 & -84 & -26 & 3285 & \text { Left Cerebellum Crus I } & 3.63\end{array}$

Good outcome < Poor outcome

\begin{tabular}{cccccc}
33 & -43 & -49 & 6136 & Right Cerebellum VIIla & 4.82 \\
-32 & -53 & -54 & 3938 & Left Cerebellum VIIla & 4.08 \\
-8 & -84 & -23 & 560 & Left Cerebellum Crus I & 3.63 \\
\hline \multicolumn{7}{l}{ GMV gray matter volume, HC healthy controls, HFM high frequency migraine }
\end{tabular}

over the bilateral cerebellum and the right precentral gyrus compared to HC (Table 2 and Fig. 1b).

\section{GMV difference between patients with HFM with poor and good outcomes}

Compared to patients with good outcome, those with poor outcome had increased GMV over the right cerebellar VIIIa, the left cerebellar VIIIa, and the left cerebellum crus I (Table 2 and Fig. 1c). This finding did not change after further controlling for disease duration or MIDAS score.

The volume of the right cerebellar VIIIa was correlated to disease duration $(r=0.34, P=0.014)$. In addition, the volumes of the right and left cerebellar VIIIa areas were correlated to 2-year headache frequencies $(r=0.38$, $P=0.005 ; r=0.35, P=0.009$, respectively), and the correlation persisted over the right cerebellar VIIIa $(r=$ $0.31, P=0.032)$ after further controlling for disease duration and MIDAS score.

Due to the arbitrary definition of $\geq 10$ days/month in HFM, we also tested the difference in GMV between the two outcome groups among patients with CM only. The results were similar in that patients with $\mathrm{CM}$ with a poor outcome had increased GMV over the right and the left cerebella (Supplement Table 2).

\section{SC networks between the three cerebellar regions and} other brain regions in patients with different outcomes We tested the interactive effect of outcome on the SC networks between the three cerebellar regions that showed volumetric difference between outcome groups 


\section{A HFM vs. HC}
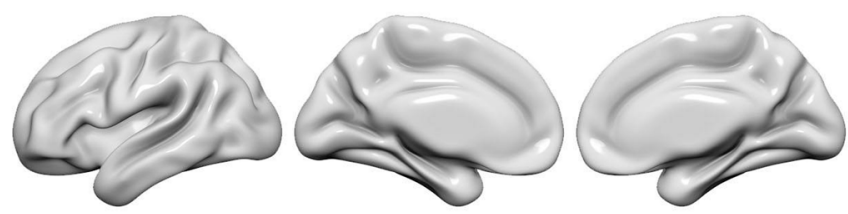

Rt. supramarginal gyrus

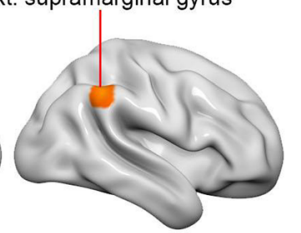

Rt cerebellum crús
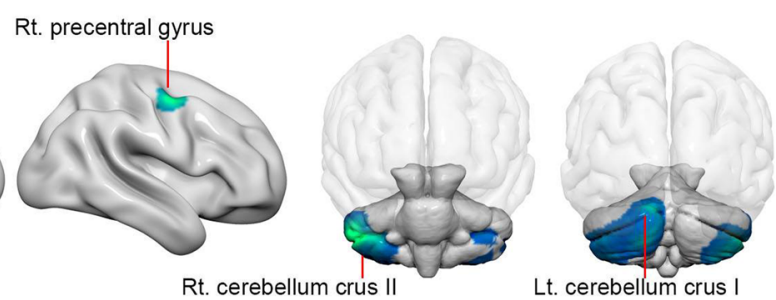

Lt. posterior cingulate gyrus
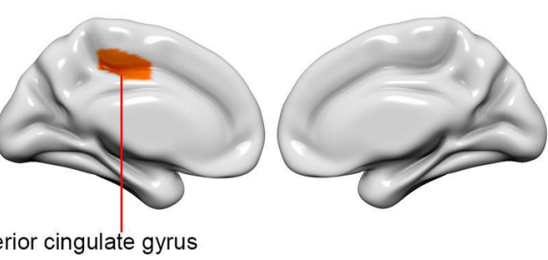

with good outcome

C

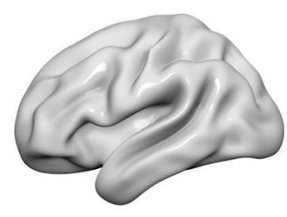

t-value

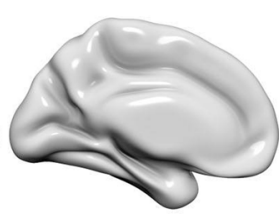

GMV decrease

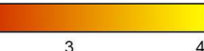

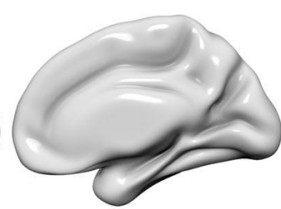

GMV increase

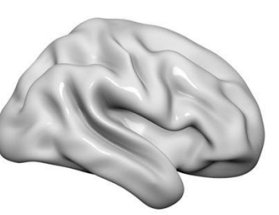

Bilateral cerebellum VIIla

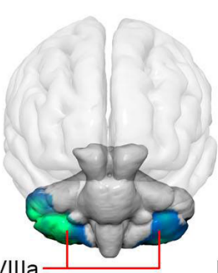

Lt. cerebellum crus I

Fig. 1 The red and blue markers are regions of different gray matter volume (a) between patients with high-frequency migraine (HFM) and healthy controls $(\mathrm{HC})$; (b) between patients with poor outcome and $\mathrm{HC}$; (c) between patients with different outcomes

and the other regions of the whole brain. In patients with poor outcome, the right cerebellar VIIIa showed an increase of SC with the right cuneus, the left cerebellar VIIIa showed an increase of SC with the right temporal gyrus, and the left cerebellum crus I showed increases of SC with the left temporal pole, left middle frontal gyrus, left calcarine cortex, and left and right cerebellum VI areas. Conversely, the SC between the left cerebellar VIIIa and the left frontal pole was decreased in patients with poor outcome (Table 3 and Fig. 2).

Further, the SC integrities between the right cerebellar VIIIa and right cuneus were correlated to 2-year headache frequencies $(r=0.36, P=0.008)$.

\section{Predictive values of cerebellar GMV and its SC integrity for headache outcomes}

In the logistic regression analysis, the GMV of the right cerebellar VIIIa and the integrity of the SC between the right cerebellar VIIIa with the right cuneus were independent predictors of poor headache outcome $(P=0.006$ and $P=0.005$, respectively). When considering only the clinical profiles (age, sex, disease duration, baseline headache frequency, and HADS and MIDAS scores), the regression model could distinguish patients with poor outcomes from those with good outcomes with fair
Table 3 Difference in structural covariance between patients with HFM with different outcomes

\begin{tabular}{llll}
\hline MNI coordinates & $\begin{array}{l}\text { Voxel } \\
\text { size }\end{array}$ & Anatomical region & $\begin{array}{l}\text { Local peak } \\
\text { T-value }\end{array}$ \\
\hline
\end{tabular}

Seed 1: Right cerebellum VIlla

Poor outcome $>$ Good outcome

$\begin{array}{lllll}14 & -84 & 38 & 272 & \text { Right cuneus }\end{array}$

Seed 2: Left cerebellum VIIla

Poor outcome $>$ Good outcome

$\begin{array}{lllll}56 & -13 & -23 & 325 & \text { Right middle temporal gyrus } 3.64\end{array}$

Poor outcome $<$ Good outcome

$\begin{array}{lllll}-48 & 47 & -6 & 331 \quad \text { Left frontal pole }\end{array}$

Seed 3: Left cerebellum crus I

Poor outcome $>$ Good outcome

$\begin{array}{llllll}-38 & 23 & -39 & 557 & \text { Left temporal pole } & 4.30\end{array}$

$\begin{array}{lllll}-30 & 29 & 50 & 420 & \text { Left middle frontal gyrus } \quad 3.49\end{array}$

$\begin{array}{lllll}-12 & -72 & 9 & 292 & \text { Left calcarine cortex }\end{array} 3.49$

$\begin{array}{lllll}-39 & -45 & -30 & 918 & \text { Left cerebellum VI }\end{array}$

$\begin{array}{lllll}37 & -41 & -32 & 686 & \text { Right cerebellum Vl }\end{array}$

HFM high frequency migraine 


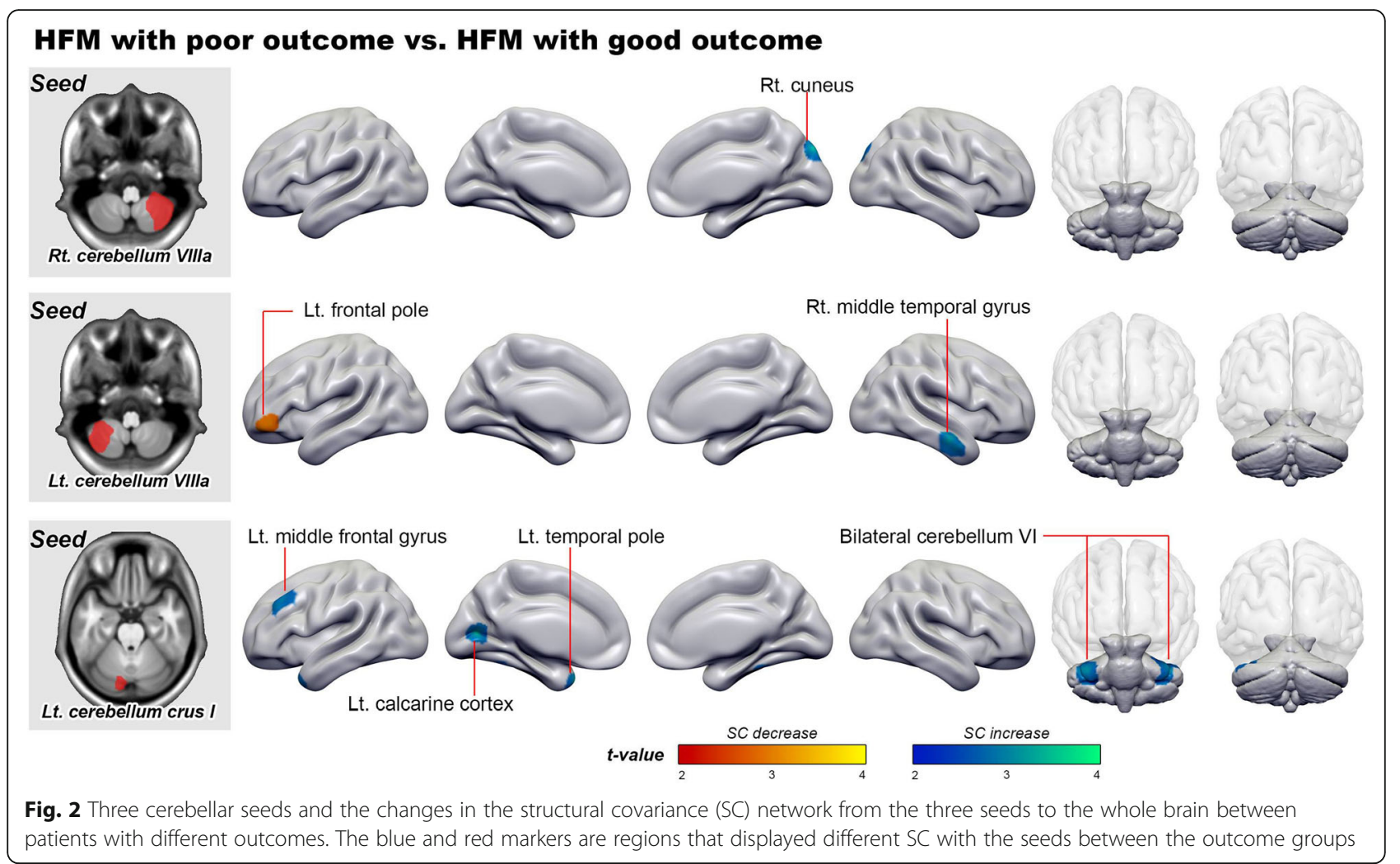

discrimination $(\mathrm{AUC}=0.72$ ). When we added the GMV data of the right cerebellar VIIIa and of the integrity of its $\mathrm{SC}$ with the right cuneus, the ability of discriminating outcomes increased from fair to good (AUC $=0.93$ ).

\section{Discussion}

The current study showed that patients with HFM, despite similar age, sex, baseline headache characteristics and psychiatric profiles, differing in neuroimaging features specifically over the posterior lobules of the bilateral cerebellar hemisphere had different 2-year headache outcomes.

In recent years, mounting evidence has shown that the cerebellum is not only involved in motor and coordinative functions, but is also involved in emotion, cognition, learning [30, 31]. In addition, cerebellum has been demonstrated to respond to noxious stimuli and involve pain processing [31-34], but the specific function of cerebellum during pain processing and its role in pain disorder is not clear. In migraine, cerebellar activation was demonstrated not only in trigeminal nociception but also during a migraine episode [35, 36]. Additionally, more silent ischemic lesions have been found over the cerebellum in patients with migraine with or without aura [37, 38]. These evidences link migraine pain and the consequences of repeated attacks with the cerebellum.

There were few studies reported volumetric changes of the cerebellum in patients with migraine, and the results varied with headache frequency. One study showed decreased cerebellar volume in patients with low frequency episodic migraine (mean monthly headache days $=4.7$ ) [39]. Another study enrolled most patients with CM (episodic/chronic migraine: 8/46) showed increased GMV of the right cerebellar hemisphere, and the GMV increase was associated with a lower headache frequency and a shorter disease duration [40]. Our study in patients with HFM, in accordance with the latter one, also demonstrated a volume increase of the right cerebellar hemisphere, which correlated with higher disability (the MIDAS score). Further, our 2-year follow-up revealed an unfavorable outcome in those patients with greater cerebellar volume since they were less likely to have longterm headache remission. The above findings remained the same after adjusting for the covariates of baseline headache profiles. Therefore, the cerebellum not only involves the processing of migraine pain but also plays a role in migraine prognosis.

The role of cerebellum in migraine prognosis is also supported by other findings of the present study. A greater connectivity between the cerebellum and the cuneus, the calcarine cortex, and the temporal lobe, and a lesser connectivity between the cerebellum and the prefrontal cortex were associated with a poor headache prognosis. Earlier MRI studies in migraine also demonstrated functional connectivity change between the cerebellum and other brain structures. Functional connectivity was decreased 
between the cerebellum and the thalamus, occipital areas, and fusiformis gyrus, respectively, during trigeminal nociception in patients with migraine. The authors thus suggested a decreased inhibitory control of cerebellum on gating and nociceptive processing [40]. Another study showed increased resting functional connectivity of cerebellum with prefrontal cortex in patients with episodic migraine [39]. Taken together, through interacting with other brain structures, the cerebellum may modulate pain processing and pain persistence in patient with migraine.

Few neuroimaging studies have explored brain signatures of migraine prognosis. Our previous studies have shown that the volumes of the right hippocampus and the orbitofrontal cortex are associated with headache outcomes in patients with migraine and patients with CM with medication overuse, respectively [16, 41]. The current study showed the posterior lobe of cerebellum was associated with long-term migraine prognosis, specifically in patients with HFM without medication overuse. Although the mechanism of cerebellar involvement in headache prognosis is not clear, there were evidences that the larger cerebellar volume is associated with chronic widespread body pain [42]. Further, previous fMRI studies have shown the posterior lobes of cerebellum are activated during anticipation of pain [43]. Anticipation of pain lowers the behavior performance and increases pain intensity in patients with chronic pain [44]. More importantly, fear of pain, driven by the anticipation of pain, is a prognostic factor for chronic pain [45]. Whether this phenomenon and its association with cerebellum underlie the chronicity of migraine is of interest and needs to be further studied. Previous studies have shown varied cortical excitability in patients with migraine with different headache frequencies and outcomes $[46,47]$. Further studies are required to determine if the cerebellum and its output contribute to the metaplasticity-like phenomenon at the cortical level and modulate cortical excitability in patients with migraine.

Some caveats and limitations should be addressed when interpreting the results of this study. First, to explore the brain signature related to outcome, we controlled for potential confounders that could possibly influence the GMV between the outcome groups. The GMV of the cerebellum remained significantly different between patients with different outcomes when we further controlled for disease duration and MIDAS score. However, there may be confounders that we did not identify in this study, such as musculoskeletal pain, that could potentially change brain GMV and headache prognosis. Second, the results of the current study were generated through VBM analysis. It should be borne in mind that some specific regions over the brainstem or limbic system may be too small for whole-brain analysis. The complex tissue pattern in these deep structures may also hamper the precision of VBM-based tissue segmentation. Third, the study was conducted with patients with either HFM or CM. HFM and CM are two clinical headache diagnoses arbitrarily separated by headache frequency of 10 and 15 days per month. Previous studies have shown similar symptom profiles, sociodemographic characteristics, and comorbidities between the two groups of patients [48]. These evidences support combining the two groups of patients when investigating their outcomes and neuroimage. Besides, the main study results were identical when we conducted the analysis only with patients with CM. Last, although we followed-up the clinical outcome of these patients at 2 years, we did not perform the second scan of brain MRI of these patients to see if the cerebellar volume changes are persistent. However, it should be noted that the patients with different outcomes had very different headache frequencies at 2-year followup, and their psychiatric conditions and the impact of their headaches may also vary with time. All these factors can cause unwanted confounding that hinders our identification of the brain signature predictive of headache outcome in patients with similar clinical profiles.

\section{Conclusion}

Structural and connectivity changes of the cerebellum are associated with persistence or progression of headache frequency in patients with migraine with highly frequent attacks at baseline. Further studies are required to elucidate the role of the cerebellum in migraine chronification and investigate the potential role of the cerebellum in the treatment of refractory CM.

\section{Supplementary information}

Supplementary information accompanies this paper at https://doi.org/10. 1186/s10194-020-01096-4.

Additional file 1.Table 1. Demographics and clinical profile of patients with high frequency episodic migraine and chronic migraine.

Additional file 2. Table 2. Difference in GMV between patients with CM with different outcomes.

\section{Abbreviations}

HC: Healthy controls; HFM: High frequency migraine; CM: Chronic migraine; NRS: Numeric rating scale; TIV: Total intracranial volume; GMV: Gray matter volume; WM: White matter; VBM: Voxel based morphometry; SC: Structural covariance; MIDAS: Migraine Disability Assessment; HADS-A: The anxiety subscale of the Hospital Anxiety and Depression Scale; HADS-D: The depression subscale of the Hospital Anxiety and Depression Scale

\section{Acknowledgements}

We would like to thank the study participants and the research assistants for actively participating.

\section{Authors' contributions}

HY Liu: drafting initial manuscript, manuscript revision, study design, data acquisition, data analysis; PL Lee and KH Chou: data analysis and manuscript revision; KL Lai, YF Wang, and SP Chen: data acquisition; WT Chen and SJ Wang: study design, data acquisition, and manuscript revision. The authors read and approved the final manuscript. 


\section{Funding}

This work was supported by grants from Ministry of Science and Technology of Taiwan (107-2314-B-075-058-MY3 to HY Liu, 106-2221-E-010-011- and 107-2221-E-010-010-MY3 to KH Chou, 107-2314-B-075 -015 -MY2 to WT Chen, 108-2321-B-010-014-MY2, 108-2321-B-010-001- and 108-2314-B-010023-MY3 to SJ Wang), Taipei Veterans General Hospital (V108C-129 to WT Chen), Ministry of Health and Welfare, Taiwan (108-TDU-B-211-133001), and Brain Research Center, National Yang-Ming University from The Featured Areas Research Center Program within the framework of the Higher Education Sprout Project by the Ministry of Education in Taiwan.

\section{Availability of data and materials}

Anonymized and statistical information of all the participants was made available to and shared only among qualified investigators.

\section{Ethics approval and consent to participate}

All participants completed informed consent forms after receiving a complete explanation of the study. The Institutional Review Board of Taipei Veterans General Hospital approved the study protocol.

\section{Consent for publication}

Not applicable.

\section{Competing interests}

HY Liu, PL Lee, KH Chou, KL Lai, YF Wang, SP Chen, and WT Chen have no conflict of interest to declare.

SJ Wang has served on the advisory boards of Eli Lilly, Daiichi-Sankyo, Taiwan Pfizer and Taiwan Norvatis. He has received honoraria as a moderator from Allergan, Pfizer, Eli Lilly, Bayer, and Eisai.

\section{Author details}

'Department of Neurology, Neurological Institute, Taipei Veterans General Hospital, No. 201, Sec. 2 Shih-Pai Rd, Taipei, Taiwan. ${ }^{2}$ School of Medicine, National Yang-Ming University, Taipei, Taiwan. ${ }^{3}$ Institute of Neuroscience, National Yang-Ming University, Taipei, Taiwan. ${ }^{4}$ Brain Research Center, National Yang-Ming University, Taipei, Taiwan. ${ }^{5}$ Division of Translational Research, Department of Medical Research, Taipei Veterans General Hospital, Taipei, Taiwan.

\section{Received: 25 January 2020 Accepted: 9 March 2020} Published online: 18 March 2020

\section{References}

1. Bigal ME, Serrano D, Buse D, Scher A, Stewart WF, Lipton RB (2008) Acute migraine medications and evolution from episodic to chronic migraine: a longitudinal population-based study. Headache 48(8):1157-1168

2. Hagen K, Kristoffersen ES, Winsvold BS, Stovner L, Zwart J-A (2018) Remission of chronic headache: an 11-year follow-up study. Data from the Nord-Trøndelag health surveys 1995-1997 and 2006-2008. Cephalalgia 38(14):2026-2034

3. Bigal ME, Lipton RB (2008) Clinical course in migraine: conceptualizing migraine transformation. Neurology 71(11):848-855

4. Manack AN, Buse DC, Lipton RB (2011) Chronic migraine: epidemiology and disease burden. Curr Pain Headache Rep 15(1):70-78

5. Lipton RB (2009) Tracing transformation. Neurology 72(5 Supplement 1):S3

6. Buse DC, Greisman JD, Baigi K, Lipton RB (2019) Migraine progression: a systematic review. Headache 59(3):306-338

7. Schwedt TJ, Schlaggar BL, Mar S, Nolan T, Coalson RS, Nardos B et al (2013) Atypical resting-state functional connectivity of affective pain regions in chronic migraine. Headache 53(5):737-751

8. Valfre W, Rainero I, Bergui M, Pinessi L (2008) Voxel-based morphometry reveals gray matter abnormalities in migraine. Headache 48(1):109-117

9. Schwedt TJ, Dodick DW (2009) Advanced neuroimaging of migraine. Lancet Neurol 8(6):560-568

10. Katsarava Z, Schneeweiss S, Kurth T, Kroener U, Fritsche G, Eikermann A et a (2004) Incidence and predictors for chronicity of headache in patients with episodic migraine. Neurology 62(5):788

11. Torres-Ferrús M, Quintana M, Fernandez-Morales J, Alvarez-Sabin J, PozoRosich P (2016) When does chronic migraine strike? A clinical comparison of migraine according to the headache days suffered per month. Cephalalgia 37(2):104-113
12. Bigal ME, Lipton RB (2008) Clinical course in migraine. Neurology 71(11):848

13. Headache Classification Committee of the International Headache Society (IHS) (2018) The International Classification of Headache Disorders 3rd Edition. Cephalalgia 38(1):1-211

14. Juang KD, Wang SJ, Lin CH, Fuh JL (1999) Use of the hospital anxiety and depression scale as a screening tool for patients with headache. Zhonghua yi xue za zhi = Chinese medical journal. Free China ed 62(11):749-755

15. Stewart WF, Lipton RB, Whyte J, Dowson A, Kolodner K, Liberman JN et al (1999) An international study to assess reliability of the migraine disability assessment (MIDAS) score. Neurology 53(5):988-994

16. Liu HY, Chou KH, Lee PL, Fuh JL, Niddam DM, Lai KL, et al (2017) Hippocampus and amygdala volume in relation to migraine frequency and prognosis. Cephalalgia 37(14):1329-1336

17. Ashburner J, Friston KJ (2000) Voxel-based Morphometry-the methods. Neurolmage 11(6):805-821

18. Lorio S, Fresard S, Adaszewski S, Kherif F, Chowdhury R, Frackowiak RS et al (2016) New tissue priors for improved automated classification of subcortical brain structures on MRI. Neurolmage 130:157-166

19. Ashburner J (2007) A fast diffeomorphic image registration algorithm. Neurolmage 38(1):95-113

20. Johnson WE, Li C, Rabinovic A (2006) Adjusting batch effects in microarray expression data using empirical Bayes methods. Biostatistics 8(1):118-127

21. Fortin J-P, Parker D, Tunç B, Watanabe T, Elliott MA, Ruparel K et al (2017) Harmonization of multi-site diffusion tensor imaging data. Neurolmage 161: 149-170

22. Fortin J-P, Cullen N, Sheline Yl, Taylor WD, Aselcioglu I, Cook PA et al (2018) Harmonization of cortical thickness measurements across scanners and sites. Neurolmage 167:104-120

23. Yu M, Linn KA, Cook PA, Phillips ML, McInnis M, Fava M et al (2018) Statistical harmonization corrects site effects in functional connectivity measurements from multi-site fMRI data. Hum Brain Mapp 39(11):4213-4227

24. Alexander-Bloch A, Giedd JN, Bullmore E (2013) Imaging structural covariance between human brain regions. Nat Rev Neurosci 14(5):322-336

25. Mechelli A, Friston KJ, Frackowiak RS, Price CJ (2005) Structural covariance in the human cortex. J Neurosci 25(36):8303-8310

26. Romero-Garcia R, Whitaker KJ, Vasa F, Seidlitz J, Shinn M, Fonagy P et al (2018) Structural covariance networks are coupled to expression of genes enriched in supragranular layers of the human cortex. Neurolmage 171:256-267

27. Seeley WW, Crawford RK, Zhou J, Miller BL, Greicius MD (2009) Neurodegenerative diseases target large-scale human brain networks. Neuron 62(1):42-52

28. Chou K-H, Lin W-C, Lee P-L, Tsai N-W, Huang Y-C, Chen H-L et al (2015) Structural covariance networks of striatum subdivision in patients with Parkinson's disease. Hum Brain Mapp 36(4):1567-1584

29. Eisenberg IW, Wallace GL, Kenworthy L, Gotts SJ, Martin A (2015) Insistence on sameness relates to increased covariance of gray matter structure in autism spectrum disorder. Molecular Autism 6(1):54

30. Timmann D, Drepper J, Frings M, Maschke M, Richter S, Gerwig M et al (2010) The human cerebellum contributes to motor, emotional and cognitive associative learning. A review. Cortex 46(7):845-857

31. Moulton EA, Schmahmann JD, Becerra L, Borsook D (2010) The cerebellum and pain: passive integrator or active participator? Brain Res Rev 65(1):14-27

32. Bocci T, Santarcangelo E, Vannini B, Torzini A, Carli G, Ferrucci R et al (2015) Cerebellar direct current stimulation modulates pain perception in humans. Restor Neurol Neurosci 33(5):597-609

33. Pereira M, Rafiq B, Chowdhury E, Babayev J, Boo H, Metwaly R et al (2017) Anodal cerebellar tDCS modulates lower extremity pain perception. NeuroRehabilitation 40(2):195-200

34. Bocci T, De Carolis G, Ferrucci R, Paroli M, Mansani F, Priori A et al (2019 Jun) Cerebellar Transcranial direct current stimulation (ctDCS) ameliorates phantom limb pain and non-painful phantom limb sensations. Cerebellum 18(3):527-535

35. Kros L, Angueyra Aristizábal CA, Khodakhah K (2018) Cerebellar involvement in migraine. Cephalalgia 38(11):1782-1791

36. Mehnert J, Schulte L, Timmann D, May A (2017) Activity and connectivity of the cerebellum in trigeminal nociception. Neurolmage 150:112-118

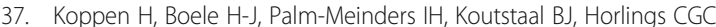
Koekkoek BK et al (2016) Cerebellar function and ischemic brain lesions in migraine patients from the general population. Cephalalgia 37(2):177-190

38. Kruit MC, van Buchem MA, Hofman PA, Bakkers JT, Terwindt GM, Ferrari MD et al (2004) Migraine as a risk factor for subclinical brain lesions. JAMA 291(4):427-434 
39. Jin C, Yuan K, Zhao L, Zhao L, Yu D, von Deneen KM et al (2013) Structural and functional abnormalities in migraine patients without aura. NMR Biomed 26(1):58-64

40. Mehnert J, May A (2017) Functional and structural alterations in the migraine cerebellum. J Cereb Blood Flow Metab 39(4):730-739

41. Lai TH, Chou KH, Fuh JL, Lee PL, Kung YC, Lin CP et al (2016) Gray matter changes related to medication overuse in patients with chronic migraine. Cephalalgia 36(14):1324-1333

42. Shi HC, Yuan CH, Dai ZY, Ma HR, Sheng LQ (2016) Gray matter abnormalities associated with fibromyalgia: a meta-analysis of voxel-based morphometric studies. Semin Arthritis Rheum 46(3):330-337

43. Stoodley CJ, Schmahmann JD (2010) Evidence for topographic organization in the cerebellum of motor control versus cognitive and affective processing. Cortex 46(7):831-844

44. Pfingsten M, Leibing E, Harter W, Kroner-Herwig B, Hempel D, Kronshage U et al (2001) Fear-avoidance behavior and anticipation of pain in patients with chronic low back pain: a randomized controlled study. Pain Med 2(4):259-266

45. Turk DC, Wilson HD (2010) Fear of pain as a prognostic factor in chronic pain: conceptual models, assessment, and treatment implications. Curr Pain Headache Rep 14(2):88-95

46. Restuccia D, Vollono C, del Piero I, Martucci L, Zanini S (2013 Sep) Different levels of cortical excitability reflect clinical fluctuations in migraine. Cephalalgia 33(12):1035-1047

47. Chen WT, Wang SJ, Fuh JL, Lin CP, Ko YC, Lin YY (2011) Persistent ictal-like visual cortical excitability in chronic migraine. Pain 152(2):254-258

48. Lipton RB, Serrano D, Pavlovic JM, Manack AN, Reed ML, Turkel CC et al (2014) Improving the classification of migraine subtypes: an empirical approach based on factor mixture models in the American Migraine Prevalence and Prevention (AMPP) study. Headache 54(5):830-849

\section{Publisher's Note}

Springer Nature remains neutral with regard to jurisdictional claims in published maps and institutional affiliations.

Ready to submit your research? Choose BMC and benefit from:

- fast, convenient online submission

- thorough peer review by experienced researchers in your field

- rapid publication on acceptance

- support for research data, including large and complex data types

- gold Open Access which fosters wider collaboration and increased citations

- maximum visibility for your research: over $100 \mathrm{M}$ website views per year

At $\mathrm{BMC}$, research is always in progress.

Learn more biomedcentral.com/submissions 